\title{
Low- $n-6$ and low-n-6 plus high-n-3 diets for use in clinical research
}

\author{
Beth A. MacIntosh ${ }^{1 *}+$, Christopher E. Ramsden ${ }^{2} \dagger$, Keturah R. Faurot ${ }^{3,4}$, Daisy Zamora ${ }^{4}$, \\ Margaret Mangan ${ }^{5}$, Joseph R. Hibbeln ${ }^{6}$ and J. Douglas Mann ${ }^{7}$ \\ ${ }^{1}$ Nutrition Research and Metabolism Core, North Carolina Translational Clinical Sciences Institute, 101 Manning Drive, \\ CB\#7600, University of North Carolina at Chapel Hill, Chapel Hill, NC 27599, USA \\ ${ }^{2}$ Section on Nutritional Neurosciences, Laboratory of Membrane Biochemistry and Biophysics, NIAAA, NIH, 31 Center Drive, \\ Building 31, Room 1B-58, Bethesda, MD 20892, USA \\ ${ }^{3}$ Department of Epidemiology, Gillings School of Global Public Health, University of North Carolina at Chapel Hill, Chapel \\ Hill, NC 27599, USA \\ ${ }^{4}$ Program on Integrative Medicine, Department of Physical Medicine and Rehabilitation, School of Medicine, University of \\ North Carolina at Chapel Hill, Chapel Hill, NC 27599, USA \\ ${ }^{5}$ Nutrition Obesity Research Center - Diet, Physical Activity and Body Composition in Human Populations, Gillings School of \\ Public Health, University of North Carolina at Chapel Hill, Chapel Hill, NC 27599, USA \\ ${ }^{6}$ Section on Nutritional Neurosciences, Laboratory of Membrane Biochemistry and Biophysics, NIAAA, NIH, 5625 Fishers \\ Lane, Room 3N-O7, Bethesda, MD 20892, USA \\ ${ }^{7}$ Department of Neurology, School of Medicine, University of North Carolina at Chapel Hill, Chapel Hill, NC 27599-7025, USA
}

(Submitted 20 April 2012 - Final revision received 16 October 2012 - Accepted 17 October 2012 - First published online 18 January 2013)

\section{Abstract}

Few trials have evaluated the metabolic effects and health outcomes of lowering dietary $n-6$ PUFA. The objectives of the present paper were (1) to report the methods employed to lower dietary $n$-6 PUFA, while either increasing or maintaining $n$ - 3 PUFA intake and (2) to validate our methods with $24 \mathrm{~h}$ recalls and erythrocyte fatty acid analyses. A total of sixty-seven subjects were randomised to either (1) an average- $n$-3 PUFA, low- $n-6$ PUFA (L6) intervention designed to lower linoleic acid (LA; $\leq 2 \cdot 5 \%$ of energy (en\%)) and arachidonic acid ( $\leq 60 \mathrm{mg} / \mathrm{d}$ ), while maintaining an average US intake of $n-3$ PUFA or (2) a high- $n$-3 PUFA, low- $n$ - 6 PUFA (H3-L6) intervention designed to lower $n$ - 6 LA, while increasing the $n$ - 3 PUFA $\alpha$-linolenic acid (ALA; $\geq 1.5$ en $\%$ ) and EPA + DHA ( $\geq 1000 \mathrm{mg} / \mathrm{d}$ ). Pre- and intraintervention nutrient intakes were estimated with six $24 \mathrm{~h}$ dietary recalls per subject. Both groups achieved the targeted reductions in dietary LA to $\leq 2.5$ en\% (median LA $2 \cdot 45(2 \cdot 1,3 \cdot 1) ; P<0 \cdot 001)$. Intakes of $n-3$ PUFA did not change for the L6 group. Target increases in $n$-3 ALA (median $1 \cdot 6$ en\%, (1·3, 2.0), $P<0 \cdot 001)$ and EPA + DHA (1482 mg, (374, 2558), $P<0 \cdot 001)$ were achieved in the H3-L6 group. Dietary changes were validated by corresponding changes in erythrocyte $n-6$ and $n-3$ fatty acid composition. Dietary LA can be lowered to $\leq 2 \cdot 5$ en\%, with or without concurrent increases in dietary $n-3$ PUFA, in an outpatient clinical trial setting using this integrated diet method.

Key words: $n$-6 Fatty acids: $n$-3 Fatty acids: Polyunsaturated fatty acids: Dietary interventions: Headache

Modern per capita US $n-6$ linoleic acid (LA) intake of 6.3-6.7\% of energy $(\mathrm{en} \%)^{(1)}$ is substantially higher than available data estimating historical US $(2 \cdot 2 \mathrm{en} \%)^{(2)}$ and evolutionary (2.3-3.6 en\%) LA consumption ${ }^{(3)}$. Since the early $1960 \mathrm{~s}$, dietary advice to substitute vegetable oils for animal fats contributed to major changes in the US food supply, including a reduction in $\mathrm{SFA}^{(1)}$ and a 3-fold increase in LA intake ${ }^{(2)}$. Data on the metabolic and health effects of these marked dietary changes are limited ${ }^{(4)}$. Dietary $n-6$ and $n-3$ PUFA are known to alter the composition and metabolic functions of various tissues in both animal models ${ }^{(5-8)}$ and human subjects ${ }^{(4,9,10)}$. Several, but not all, controlled trials comparing $n$-3 PUFA supplements $v$. placebo have demonstrated substantial health benefits ${ }^{(11-15)}$

Since $n-6$ and $n-3$ fatty acids compete to some extent for enzymatic conversion, tissue incorporation and metabolism to bioactive derivatives, lowering dietary LA could potentially

Abbreviations: AA, arachidonic acid; en $\%$, percentage of energy; H3-L6, high- $n-3$, low- $n-6$ diet; IQR, interquartile range; L6, average- $n$-3, low- $n-6$ diet; LA, linoleic acid; NDSR, Nutritional Data System for Research.

*Corresponding author: B. A. MacIntosh, fax +1919966 0576, email beth_macintosh@med.unc.edu

† Both authors contributed equally. 
augment the benefits of $n$ - 3 fatty acids or have substantial independent health benefits ${ }^{(15-17)}$. To our knowledge, no human trial has evaluated the long-term effects of lowering dietary LA from modern intakes to amounts consistent with historical US or evolutionary intakes for the prevention or management of any medical condition.

Challenges for evaluating the effects of dietary LA lowering in Western populations include: (1) the ubiquity of LA in the food supply, with LA-rich oils added to most packaged food items; (2) wide variability in the LA content of many similar food items; and (3) the abundance of LA in adipose tissue stores of Western populations. Because LA has a slow turnover rate (half-life $1-2$ years) in adipose tissue ${ }^{(18,19)}$, dietary changes may need to be maintained for relatively long periods to achieve maximal alterations in tissue composition and function.

With these unique challenges in mind, we developed a novel, integrated diet method designed to achieve marked reductions in dietary LA in an outpatient setting. Subjects with chronic daily headaches were randomised to one of two low- $n-6$ study diets: (1) an average- $n-3$, low- $n-6$ (L6) diet and (2) a high- $n-3$, low- $n-6$ (H3-L6) diet. The interventions combined four key elements: (1) provision of both unprepared and specially formulated prepared foods accounting for two-thirds of total energy intake; (2) intensive dietitianadministered counselling, with targeted and individually tailored aspects; (3) continuous self-monitoring; and (4) access to an intervention-specific website to complement and reinforce dietitian advice.

This novel dietary method was developed to achieve: (1) a reduction in $n-6$ LA intake to $\leq 2.5 \mathrm{en} \%$ in both intervention groups; and (2) an increase in $n-3 \mathrm{EPA}+\mathrm{DHA}$ to $\geq 1000 \mathrm{mg} / \mathrm{d}$ in the H3-L6 group, with no change in the L6 group. These goals were evaluated by administering six $24 \mathrm{~h}$ dietary recalls per subject. Erythrocyte fatty acids were analysed to provide further evidence of adherence. Complete metabolic and clinical headache outcomes will be presented in a separate paper.

\section{Experimental methods}

\section{Participants}

Individuals who were at least 18 years old and who satisfied the 2004 International Classification of Headache Disorders criteria for chronic daily headaches ${ }^{(20)}$ were invited to participate. Patients with chronic daily headaches, defined as at least fifteen headache days per month and $4 \mathrm{~h} / \mathrm{d}^{(21)}$, were recruited from the following sources: (1) specialty headache clinics; (2) broadcast email to the University of North Carolina (UNC) community; and (3) brochures placed in UNC medical clinics. This population with chronic and frequent pain was selected in order to evaluate the hypothesis that targeted alterations in dietary fatty acids can reduce the frequency and severity of pain outcomes. Pregnant women and those regularly consuming fatty acid supplements were excluded. The present study was conducted according to the Declaration of Helsinki guidelines, with all procedures involving human subjects approved by the UNC-Chapel Hill Institutional Review Board. All participants provided written informed consent. This trial is registered under ClinicalTrials.gov (NCT01157208).

\section{Development of study diets}

Two research diets were developed for the 12-week, parallelgroup randomised dietary intervention. The L6 diet was designed to reduce LA and arachidonic acid (AA) to $\leq 2.5$ en $\%$ and $60 \mathrm{mg} / \mathrm{d}$, respectively, while providing average US intakes of ALA and EPA + DHA. The H3-L6 diet was designed to reduce LA to $\leq 2.5 \mathrm{en} \%$, while increasing ALA and EPA + DHA. Both diets were designed to provide typical US amounts of total protein, total carbohydrate and total fat.

\section{The average-n-3, low-n-6 diet}

Lowering n-6 PUFA. LA $(18: 2 n-6)$ is the predominant PUFA in US/Western diets ${ }^{(1)}$. High-LA oils (soyabean, maize, cottonseed, safflower and sunflower) are the predominant fat sources in most US salad dressings, margarines, mayonnaises and cooking oils. LA-rich oils are also added to most packaged food items, including breads, cereals, soups and tomato sauce, as well as most snack foods such as cookies, chips (crisps) and crackers (Supplementary Appendix 1, available online).

To achieve the intervention-specific LA intake targets, study foods were carefully selected to replace those containing substantial quantities of LA (Supplementary Appendix 1, available online). The United States Department of Agriculture National Nutrient Database for Standard Reference, Release 21, was used to screen high-LA and low-LA foods for potential inclusion and exclusion from research diets. Next, to verify low LA content, the fatty acid content of multiple brands of low-LA foods was analysed by GC, as previously described ${ }^{(22)}$. The lowest-LA foods were selected for inclusion in both diets. To facilitate dietary LA lowering, participants were provided with, and instructed to exclusively use, study-provided lowLA oils and fat sources (Table 1), including coconut oil, macadamia nut oil, low-LA olive oil, butter, fat-free mayonnaise and macadamia-vinaigrette salad dressing. Because most US packaged food items contain substantial amounts of added LA, participants also received a variety of low-LA substitute foods, including crackers, tortillas, breads and popcorn. Research foods were procured and prepared by the UNC Nutrition Research and Metabolism Core of the Clinical and Translational Research Center.

Lowering dietary arachidonic acid. Foods with high AA (20:4n-6) content, according to the United States Department of Agriculture National Nutrient Database for Standard Reference, Release 21, were first identified as key items to eliminate from the research diets (Supplementary Appendix 1, available online). Fatty acid analysis was then performed on several animal protein sources in order to identify study foods containing the lowest amounts of both LA and AA (Table 1). Participants were instructed to restrict consumption of egg yolks, meat, most poultry and certain seafood. Adequate protein intake was achieved with beans, very lean (low $n$-3) seafood, turkey, egg-whites and dairy products. Examples of 
study-provided protein sources included canned beans, prepared vegetarian chili, frozen low-fat seafood, cheeses and low-fat turkey (Table 1).

Average n-3 PUFA. The average US intake of ALA (18:3n-3) is about 0.6 en $\%{ }^{(1)}$, mostly from soyabean and rapeseed oils. Because these vegetable oil sources of ALA were restricted, a small amount of ground flaxseed (concentrated source of ALA) was added to L6 diet foods to maintain the average US ALA intake. Participants had the option of consuming specially formulated muffins, granola or $3 \mathrm{~g}$ of ground flaxseed added to foods of their choice. To achieve the average US EPA + DHA intake of $0 \cdot 11 \mathrm{~g} / \mathrm{d}$, participants received small amounts of seafood with the lowest amounts of EPA + DHA and AA (Table 1).

\section{The high-n-3, low-n-6 diet}

Lowering dietary linoleic acid. H3-L6 dieters were instructed to replace all high-LA foods in their diet with the same study-provided oils, fats and other low-LA foods used in the L6 diet (Table 1).

Increasing dietary n-3 PUFA. H3-L6 subjects were instructed to consume approximately $1.5 \mathrm{en} \%$ as ALA (Table 2), mostly from ground flaxseed. Options included $14 \mathrm{~g}$ of ground flaxseed each day for addition to foods of their choice, or specially prepared granola, muffins, granola bars and/or bean dip containing flaxseed. To meet EPA + DHA intake targets, H3-L6 dieters were counselled to consume 113-230g of fatty fish or shellfish per $d$ in place of other protein sources. Fatty acid analyses

Table 1. Linoleic acid (LA), $\alpha$-linolenic acid (ALA), arachidonic acid (AA), EPA and DHA present in $100 \mathrm{~g}$ portions of study-provided foods

Table 1. Linoleic acid (LA), $\alpha$-linolenic acid (ALA), arachidonic acid (AA), EPA and DHA present in $100 \mathrm{~g}$ portions of study-provided foods

\begin{tabular}{|c|c|c|c|c|c|c|c|}
\hline & Analysis & LA (g) & ALA (g) & $\mathrm{AA}(\mathrm{mg})$ & EPA (mg) & $\mathrm{DHA}(\mathrm{mg})$ & Diet group \\
\hline \multicolumn{8}{|l|}{ Plant foods } \\
\hline Coconut oil, unrefined & SNN & 0.97 & 0.0 & NA & NA & NA & Both \\
\hline Macadamia nut oil & SNN & $3 \cdot 34$ & 0.34 & NA & NA & NA & Both \\
\hline Extra virgin olive oil (Trader Joe's brand) & SNN & $6 \cdot 21$ & 0.65 & NA & NA & NA & Both \\
\hline Macadamia nut oil vinaigrette† & FP & 0.38 & 0.04 & NA & NA & NA & Both \\
\hline Italian vinaigrette (Kraft) & SNN & $2 \cdot 72$ & 0.89 & NA & NA & NA & Both \\
\hline Fat-free mayonnaise & SNN & 1.05 & 0.15 & NA & NA & NA & Both \\
\hline Flaxseed, ground & SNN & $5 \cdot 15$ & $15 \cdot 42$ & NA & NA & NA & Both \\
\hline Popcorn, no added fat & SNN & $3 \cdot 19$ & 0.06 & NA & NA & NA & Both \\
\hline Tortilla (Tunaro's brand) & SNN & 0.84 & 0.16 & NA & NA & NA & Both \\
\hline Crackers, low fat & USDA & 0.65 & 0.04 & NA & NA & NA & Both \\
\hline Whole-wheat bread (Ninth Street Bakery) & SNN & 0.6 & 0.04 & NA & NA & NA & Both \\
\hline Granola† & $\mathrm{FP}$ & 0.54 & $1 \cdot 18$ & NA & NA & NA & L6 $\ddagger$ \\
\hline Granola with flaxseed $†$ & FP & 0.97 & $2 \cdot 12$ & NA & NA & NA & H3-L6§ \\
\hline Bean dipt & FP & 0.84 & 0.05 & NA & NA & NA & L6 \\
\hline Bean dip with flaxseed $\dagger$ & FP & 0.55 & $1 \cdot 1$ & NA & NA & NA & H3-L6 \\
\hline Muffins† & $\mathrm{FP}$ & 0.4 & 0.8 & NA & NA & NA & L6 \\
\hline Muffins with flaxseed $†$ & $\mathrm{FP}$ & 0.7 & 1.58 & NA & NA & NA & H3-L6 \\
\hline Flaxseed granola bars $(1) \dagger$ & $\mathrm{FP}$ & 0.8 & $1 \cdot 0$ & NA & NA & NA & H3-L6 \\
\hline Mozzarella cheese string & USDA & 0.34 & 0.14 & NA & NA & NA & Both \\
\hline Blueberries, frozen & USDA & 0.17 & 0.11 & NA & NA & NA & Both \\
\hline Vegetarian chili† & FP & 0.14 & 0.05 & NA & NA & NA & Both \\
\hline Garbanzo beans & USDA & 0.49 & 0.02 & NA & NA & NA & Both \\
\hline Black beans & USDA & 0.13 & 0.11 & NA & NA & NA & Both \\
\hline Kidney beans & USDA & 0.11 & 0.08 & NA & NA & NA & Both \\
\hline Mixed vegetables, frozen & USDA & 0.05 & 0.02 & NA & NA & NA & Both \\
\hline Mixed fruit, frozen & USDA & 0.02 & 0.01 & NA & NA & NA & Both \\
\hline \multicolumn{8}{|l|}{ Animal foods } \\
\hline Butter, salted & SNN & 2.98 & 0.38 & 0.18 & 0.04 & 0.04 & Both \\
\hline Yogurt, low fat & USDA & 0.02 & 0.01 & NA & NA & NA & Both \\
\hline Cod, filet & SNN & 0.01 & 0.0 & 0.01 & 0.05 & 0.12 & L6 \\
\hline Tuna, low-fat chunk light & SNN & 0.01 & 0.0 & 0.03 & 0.02 & 0.18 & L6 \\
\hline Grouper, filet & SNN & 0.0 & $0 \cdot 0$ & 0.02 & 0.01 & 0.13 & L6 \\
\hline Shrimp & SNN & 0.05 & 0.0 & 0.01 & 0.05 & 0.07 & Both \\
\hline Turkey, low fat & SNN & 0.15 & 0.02 & 0.05 & 0.0 & 0.01 & L6 \\
\hline Wild salmon, filet & SNN & 0.02 & 0.01 & 0.01 & 0.14 & 0.43 & H3-L6 \\
\hline Wild albacore tuna, canned (Vital choice brand) & SNN & 0.21 & 0.13 & 0.13 & $1 \cdot 0$ & $3 \cdot 15$ & H3-L6 \\
\hline Wild salmon, canned (Vital choice brand) & SNN & 0.09 & 0.06 & 0.03 & 0.49 & 0.81 & H3-L6 \\
\hline Trout, filet & SNN & 0.07 & 0.01 & 0.02 & 0.09 & 0.34 & H3-L6 \\
\hline Swordfish, filet & SNN & 0.01 & 0.0 & 0.04 & 0.02 & 0.25 & H3-L6 \\
\hline Tuna, steaks & SNN & 0.01 & $0 \cdot 0$ & 0.04 & 0.03 & 0.24 & H3-L6 \\
\hline
\end{tabular}

SNN, Section on Nutritional Neurosciences, Laboratory of Membrane Biochemistry and Biophysics, National Institute on Alcohol Abuse and Alcoholism (NIAAA), National Institutes of Health; NA, not applicable; FP, ESHA Food Processor 10.6.0.0 (analyses labelled FP used a combination of United States Department of Agriculture (USDA) and NIAAA data); USDA, USDA Nutrient Database for Standard Reference, version 21.

* USDA SR21 nutrient values were used for diet calculations for foods with low absolute amounts and variability of $n$ - 6 LA. ESHA Food Processor 10.6.0.0 software was used to analyse study-provided recipes. To account for differences between study-provided foods and software values, values from direct fatty acid analysis of study foods were added to the database.

$\dagger$ Indicates specially formulated foods supplied via the metabolic kitchen.

¥ L6: average- $n-3$, low- $n-6$ diet.

$\S$ H3-L6: high- $n-3$, low- $n-6$ diet. 
Table 2. Demographics

(Number of subjects and percentages; mean values and standard deviations)

\begin{tabular}{|c|c|c|c|c|c|c|}
\hline & \multicolumn{2}{|c|}{$\begin{array}{c}\text { Total } \\
\text { sample } \\
(n 67)\end{array}$} & \multicolumn{2}{|c|}{$\begin{array}{l}\text { L6 diet } \\
\text { (n 34) }\end{array}$} & \multicolumn{2}{|c|}{$\begin{array}{l}\text { H3-L6 diet } \\
\text { (n 33) }\end{array}$} \\
\hline & $n$ & $\%$ & $n$ & $\%$ & $n$ & $\%$ \\
\hline \multicolumn{7}{|l|}{ Sex } \\
\hline Male & 9 & $13 \cdot 4$ & 4 & $11 \cdot 6$ & 5 & $15 \cdot 2$ \\
\hline Female & 58 & $84 \cdot 6$ & 30 & $88 \cdot 2$ & 25 & $80 \cdot 6$ \\
\hline \multicolumn{7}{|l|}{ Race/ethnicity } \\
\hline White & 58 & $86 \cdot 6$ & 30 & $88 \cdot 1$ & 28 & $84 \cdot 8$ \\
\hline African-American & 7 & $10 \cdot 5$ & 3 & $8 \cdot 8$ & 4 & $12 \cdot 1$ \\
\hline American Indian & 2 & $3 \cdot 0$ & 1 & $3 \cdot 0$ & 1 & $3 \cdot 2$ \\
\hline \multicolumn{7}{|l|}{ Marital status } \\
\hline Married & 38 & $56 \cdot 7$ & 19 & $55 \cdot 9$ & 19 & 57.5 \\
\hline Partnered & 9 & $13 \cdot 4$ & 5 & $14 \cdot 7$ & 4 & $12 \cdot 1$ \\
\hline Single & 13 & $19 \cdot 4$ & 6 & $17 \cdot 6$ & 7 & $21 \cdot 2$ \\
\hline Widowed, divorced & 7 & $10 \cdot 4$ & 4 & $11 \cdot 8$ & 3 & $9 \cdot 1$ \\
\hline \multicolumn{7}{|l|}{ Education } \\
\hline High school & 4 & $6 \cdot 1$ & 2 & $6 \cdot 2$ & 2 & $6 \cdot 1$ \\
\hline Some college & 16 & $24 \cdot 2$ & 6 & $18 \cdot 7$ & 10 & $30 \cdot 3$ \\
\hline Bachelor's degree & 19 & $29 \cdot 2$ & 11 & $34 \cdot 4$ & 8 & $24 \cdot 2$ \\
\hline Master's or higher & 26 & $40 \cdot 0$ & 13 & $39 \cdot 4$ & 13 & $40 \cdot 6$ \\
\hline \multicolumn{7}{|l|}{ Employment } \\
\hline Employed & 44 & $66 \cdot 7$ & 21 & $63 \cdot 6$ & 23 & $69 \cdot 7$ \\
\hline Student & 5 & $7 \cdot 6$ & 2 & $6 \cdot 1$ & 3 & $9 \cdot 1$ \\
\hline Retired/caretaker & 6 & $9 \cdot 1$ & 3 & $9 \cdot 1$ & 3 & $9 \cdot 1$ \\
\hline Disabled/unemployed & 11 & $16 \cdot 7$ & 7 & $20 \cdot 0$ & 4 & $12 \cdot 1$ \\
\hline \multicolumn{7}{|l|}{ Age (years) } \\
\hline \multicolumn{7}{|l|}{ Range: $18 \cdot 7-62.4$} \\
\hline Mean & \multicolumn{2}{|c|}{$41 \cdot 6$} & \multicolumn{2}{|c|}{$42 \cdot 5$} & \multicolumn{2}{|c|}{$41 \cdot 0$} \\
\hline SD & \multicolumn{2}{|c|}{11.5} & \multicolumn{2}{|c|}{$11 \cdot 0$} & \multicolumn{2}{|c|}{$12 \cdot 3$} \\
\hline
\end{tabular}

L6, average- $n-3$, low- $n-6$ diet; H3-L6, high- $n-3$, low- $n-6$ diet.

were performed on numerous types and brands of seafood to ensure selection of those with the highest EPA + DHA and low amounts of LA and AA (Table 1). The study-provided foods included frozen wild salmon fillets, trout fillets, tuna steaks, canned high- $n-3$ albacore tuna, canned high- $n-3$ wild salmon and sardines. The small amount of AA present in study-provided seafood resulted in daily AA consumption comparable with typical US diets (Table 2).

\section{Dietary intervention}

Dietitian counselling and provision of study foods. The dietitian met with each study participant for $2 \mathrm{~h}$ at the initial visit to administer a diet assessment tool, provide tailored dietary advice, review all diet education materials and provide a 2-week supply of food. Participants attended six 45-min follow-up diet counselling sessions at 2-week intervals throughout the 12-week intervention period. At each followup session, participants selected a 2-week supply of prepared and unprepared study foods (sufficient for approximately twothirds of their energy needs) from a fixed food list (Table 1). Study foods were prepared by the UNC Metabolic Kitchen and packed into 57 litre rolling coolers with ice packs for easy transport.

Self-monitoring. Participants completed a $3 \mathrm{~d}$ food record for the second study visit and a daily food checklist for all subsequent visits. The daily food checklist imposed less participant burden than food records, while still contributing to dietary adherence through daily self-monitoring of consumption of key study foods, such as fish, flaxseed and oils. Each week, the subjects rated their adherence to the diet for the week on a scale of 1 ('excellent') to 5 ('poor'). This subjective score allowed the participant to reflect on and report difficulties in following the diet and helped the dietitian address individual challenges to dietary adherence.

Web-based diet education materials. The following intervention-specific education materials were available to the participants through a password-protected website developed by the Nutrition Applications for Health Communications and Interventions Core of the UNC School of Public Health Nutrition Obesity Research Center: (1) Diet Guidelines; (2) Food Lists; (3) Seven-day Meal Plan; (4) Reading Food Labels Guide; (5) Grocery Shopping Guides; and (6) Dining Out Guide. In addition, more than fifty recipes using studyprovided foods and/or meeting study guidelines were included on the intervention website.

The diet guidelines informed participants of specific dietary changes required. The food list complemented the diet guidelines by categorising more than 270 foods into 'allowed', 'limit' and 'avoid' for easy reference. The $7 \mathrm{~d}$ meal plans provided practical guidance to achieve the nutrient targets and were assigned to each participant according to their baseline BMI and calculated for weight maintenance (example meal plan in Supplementary Appendix 2, available online).

'Reading food labels' is an activity booklet that illustrated how to identify high-LA foods by reading food ingredients lists. Grocery shopping guides, which list low-LA brandname foods in all major food categories at ten local grocery stores, assisted participants in obtaining low-LA foods for meals prepared at home. The dining out guide assisted in identification of low-LA foods at local restaurants.

\section{Assessment of diet adherence}

Multiple $24 \mathrm{~h}$ dietary recall assessment. The UNC Nutrition and Obesity Research Center Diet, Physical Activity and Body Composition in Human Populations Core (NORC) collected participant dietary intake data using six unannounced telephone-administered $24 \mathrm{~h}$ recalls per subject: three were administered prior to the intervention (two weekdays, one weekend day) and three were administered in the final 6 weeks of the 12-week intervention (two weekdays, one weekend day). Nutrient values were estimated using the Nutritional Data System for Research (NDSR) software version 2009-2010, developed by the Nutrition Coordinating Center, University of Minnesota, Minneapolis, $\mathrm{MN}^{(23)}$. Because the study-provided foods contained markedly different LA, ALA, AA, EPA and DHA contents than commonly available US foods, the fatty acid contents of study-provided foods were reconciled with the NDSR database in order to improve accuracy of the diet recalls. This required creation of custom user-recipe projects matched to one-tenth of the analysed, study-provided foods. An effort was also made to reconcile the fatty acid content of many of the foods consumed outside the home by calling local eateries. Despite these efforts, the 
LA content of some of the low-LA foods recommended for grocery shopping and dining out were not able to be reconciled with the NDSR database.

Erythrocyte fatty acid analysis. Erythrocytes were obtained from the EDTA blood samples after the plasma and buffy coat were removed. Following Bligh/Dyer extraction ${ }^{(23)}$, erythrocyte aliquots were heated at $100^{\circ} \mathrm{C}$ for $1 \mathrm{~h}$ with methanol containing $14 \%$ boron trifluoride to generate fatty acid methyl esters. These were then extracted into hexane and analysed with a flame ionisation detector gas chromatograph (Agilent 6890 Plus LAN; Agilent Technologies) equipped with a 30-m DBFFAP (nitroterephthalic acid modified polyethylene glycol) capillary column. Fatty acids were identified through comparison with a standard fatty acid methyl ester mixture (GLC-462). Values are expressed as percentages of total erythrocyte fatty acids.

\section{Data analysis}

Because of the small sample size and non-normal distributions of dietary intake variables and erythrocyte fatty acids, nonparametric analyses were performed. Median values and interquartile ranges (IQR) for targeted fatty acids were calculated for both groups. Pre-to-intra-intervention and between-group comparisons were made with the Wilcoxon signed-rank test for matched pairs and the Wilcoxon rank-sum test, respectively. $P$ values $<0.05$ were considered significant.

\section{Results}

A total of sixty-seven subjects were randomised to either the H3-L6 diet ( $n$ 33) or the L6 diet ( $n$ 34) (Table 2). Of these, eleven participants dropped out of the intervention after randomisation (four in the H3-L6 diet group, seven in the L6 diet group) due to trauma unrelated to diet $(n 1)$, personal conflicts ( $n$ 2), disqualification after randomisation (pregnancy or diabetes diagnosis) ( $n$ 3) or dislike of the assigned diet ( $n$ 5). One subject who completed all other aspects of the study could not be reached by telephone to complete the intra-intervention $24 \mathrm{~h}$ recall assessment. The non-completers ( $n$ 11) and completers with pre- and intra-intervention diet data $(n 55)$ had no significant differences in baseline intakes of any target nutrients ( $P>0.05$; Table 3 ).

Median nutrient intakes and IQR for the fifty-five participants with the pre- and intra-intervention 24-h recall data ( $n 28$ for L6 diet, $n 27$ for H3-L6 diet) are presented in Table 3. There were no significant pre-to-post intervention changes in the consumption of total fat, carbohydrate, protein or total energy in either diet group. For the L6 group, dietary LA declined from $7 \cdot 4 \mathrm{en} \%$ (IQR 5.7-9.6) to $2 \cdot 4 \mathrm{en} \%$ (IQR 2.0-2.9). The between-subject variability of dietary LA intake by diet group is illustrated in Fig. 1. Total PUFA declined from $8 \cdot 1$ en\% (IQR 6.4-10.6) to $3 \cdot 5$ en\% (IQR 3.14.3) and SFA increased from $10 \cdot 5$ en\% (IQR 9.1-11.9) to $14 \cdot 0$ en $\%$ (IQR $12 \cdot 1-17 \cdot 2$ ). Intakes of $n$-3 PUFA did not change for the L6 group. For the H3-L6 diet group, LA declined from 6.4 en $\%$ (IQR 5.3-7.4) to 2.5 en\% (IQR 2.2-3.9). ALA increased from $0.6 \mathrm{en} \%$ (IQR $0.5-0.9$ ) to $1.6 \mathrm{en} \%$

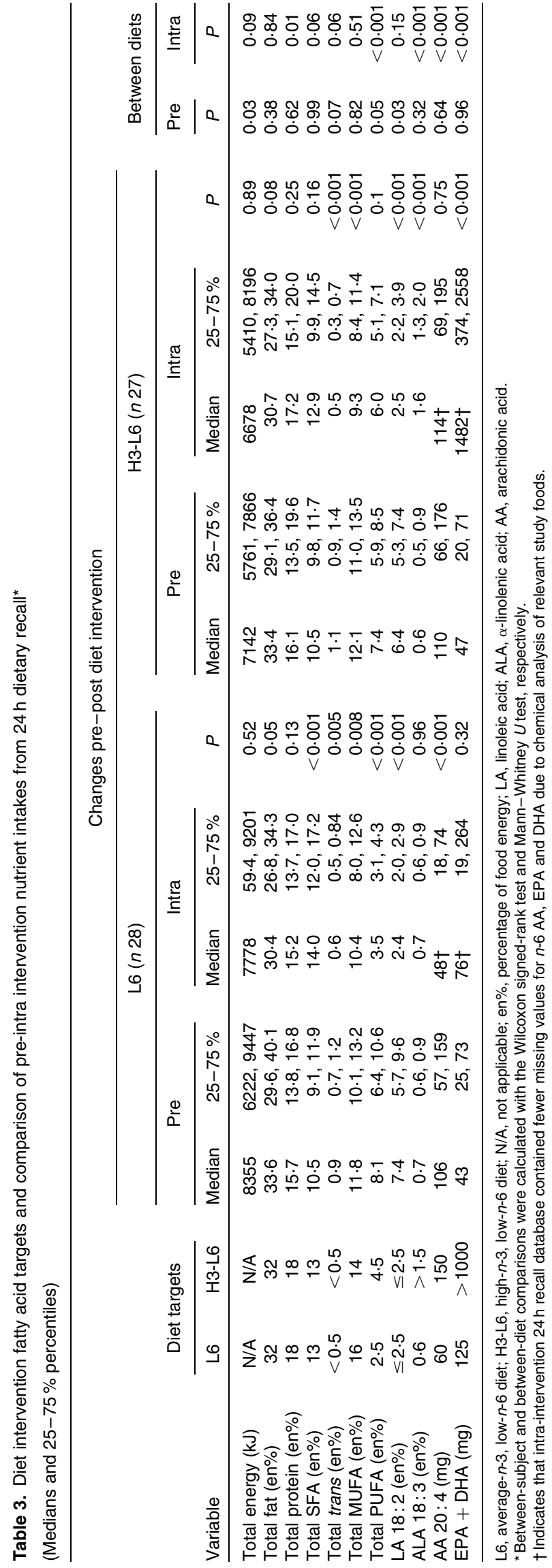




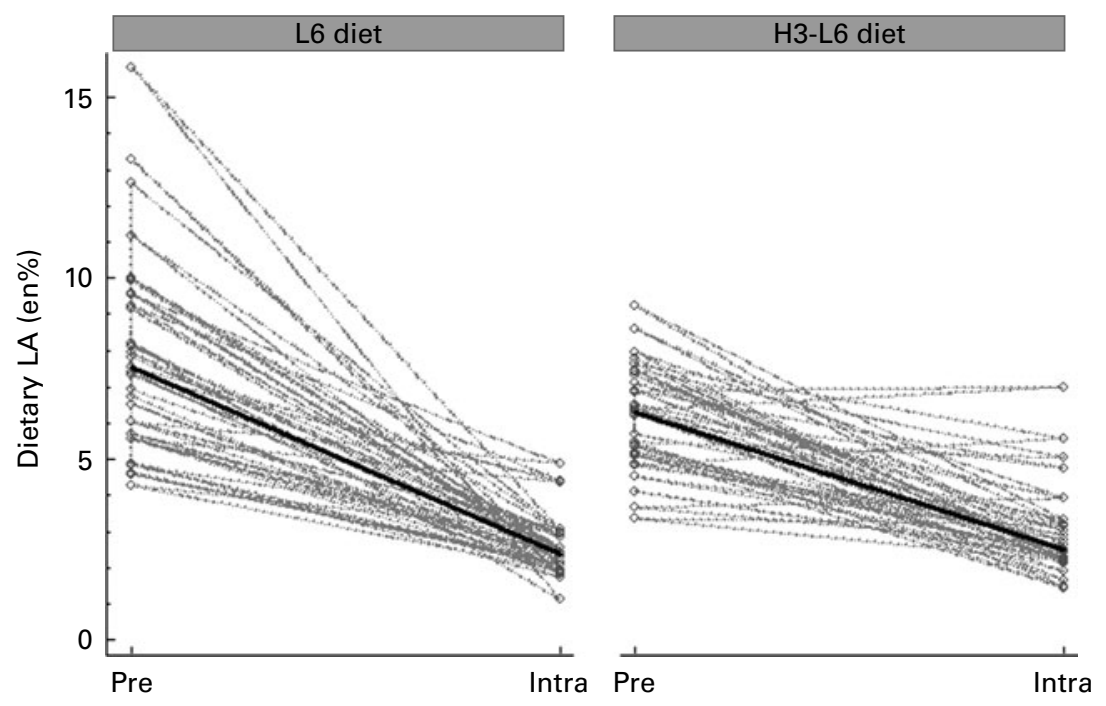

Fig. 1. Dietary linoleic acid (LA) in the pre-intervention (Pre) and intra-intervention (Intra) periods. L6, low- $n-6$ diet; H3-L6, high- $n-3$ low- $n-6$ diet; en\%, percentage of food energy. -, Median dietary LA intake.

(IQR 1·3-2·0). Daily EPA + DHA consumption increased from $47 \mathrm{mg}$ (IQR 20-71) to $1482 \mathrm{mg}$ (IQR 374-2558).

Median erythrocyte fatty acids and IQR for the fifty-two participants with pre- and post-intervention data ( $n$ 27 for L6 diet, $n 25$ for H3-L6 diet) are presented in Table 4 . For the L6 group, erythrocyte LA declined $(-13.6 \%, P<0.01)$, and EPA $(+51.3 \%, P<0.01)$, DHA $(+18.8 \%, P<0.01)$ and total SFA $(+3.0 \%, P=0.04)$ increased. For the H3-L6 diet group, LA $(-12.6 \%, P<0.01)$ and AA $(-14.1 \%, P<0.01)$ declined, and EPA $(+274 \%, P<0.01)$ and DHA $(+80 \%, P<0 \cdot 01)$ increased. Substantial within-group variability in EPA and DHA intakes were noted (Fig. 2).

\section{Discussion}

Here, we showed that dietary LA can be markedly reduced, with or without a concurrent increase in $n$-3 PUFA, in a free-living adult population using an integrated diet method. To our knowledge, this is the first demonstration that dietary $n$-6 LA can be lowered to amounts consistent with historical $\mathrm{US}^{(2)}$ or evolutionary diets ${ }^{(3)}$ in an outpatient setting.

Dietary n-6 PUFA lowering for 12-weeks reduced the LA content and increased the EPA and DHA content of the erythrocytes, without significantly altering the proportions of $n-6$ AA. Although it is often speculated that high n-6 LA intakes induce inflammation by increasing the synthesis and subsequent metabolism of AA into inflammatory derivatives ${ }^{(9,24)}$, there are currently no human data supporting the proposition that dietary LA substantially alters the abundance or metabolism of AA in human subjects ${ }^{(25)}$. The present finding that concurrent dietary lowering of both $n-6$ LA and AA did not significantly reduce the AA content of erythrocytes is consistent with the idea that dietary LA has little or no effect on tissue AA content. Importantly, however, the 12-week duration of dietary LA-lowering employed here may not have been sufficient to achieve the steady-state concentrations of erythrocyte AA due to the high LA content of the adipose tissue; LA presently accounts for about $15 \%$ of total fatty acids in the adipose tissue of the US population ${ }^{(26)}$, compared with only $6 \%$ in $1961^{(27,28)}$. Because the adipose tissue PUFA have a slow rate of turnover ${ }^{(29,30)}$ and mobilisation of LA from adipose tissue may attenuate changes in circulating PUFA, dietary LA lowering may need to be maintained for relatively long periods of time to elicit maximal changes in the AA content of the erythrocytes and other circulating lipid pools.

Dietary n-6 PUFA lowering for 12 weeks did produce significant increases in the EPA $(+51 \%)$ and DHA (+19\%) content of the erythrocytes, suggesting that high-LA diets may interfere with the synthesis and/or accumulation of EPA and DHA in human tissues. Dietary LA (18:2n-6) may reduce EPA and DHA in human tissues by: (1) impairing enzymatic conversion of $n-3$ ALA $(18: 3 n-3)$ to $18: 4 n-3$ (the precursor to EPA) ${ }^{(31)}$; (2) impairing conversion of $24: 5 n-3$ to $24: 6 n-3^{(32)}$ (the precursor to DHA) ${ }^{(33)}$; and (3) competing with $n-3$ EPA and DHA (and $n-6$ AA) for esterification into the $s n-2$ position of phospholipids ${ }^{(34)}$. It is generally believed that the synthesis of DHA from dietary ALA is not sufficient to maintain adequate DHA in human subjects ${ }^{(35)}$. The present finding of increased EPA and DHA accumulation with dietary $n$-6 lowering indicates that the DHA adequacy of human tissues may depend on background $n-6$ PUFA consumption. Importantly, however, erythrocyte fatty acid data cannot inform whether observed increases in EPA and DHA are due to increased elongation/desaturation of $n-3$ ALA, reduced competition with n-6 LA (and n-6 AA) for esterification or a combination of both. Tracer studies comparing the kinetics of n-3 ALA elongation/desaturation in high- and low-LA diets are warranted.

The H3-L6 diet produced marked increases in erythrocyte $n-3$ EPA and DHA and a reduction in n-6 AA, compared with the L6 diet; these changes were probably attributable to consumption of preformed EPA and DHA from the seafood. The observed reduction in erythrocyte AA may be due to 
increased competition with $n-3$ EPA and DHA for esterification into the phospholipid sn-2 position, reduced elongation/ desaturation of $n-6$ LA or a combination of both.

Although the H3-L6 group achieved the median EPA + DHA intake target of $\geq 1000 \mathrm{mg} / \mathrm{d}$ (Fig. 1), there was considerable within-group variability that was probably attributable to aversion to fish in a subset of the participants. H3-L6 dieters who were unwilling to consume at least one serving of seafood per d were encouraged to consume high-EPA + DHA fish 'as often as possible' and to continue all other aspects of the intervention. The use of only seafood, rather than $n-3$ supplements, to deliver EPA + DHA is a unique aspect of the present study. Some of the EPA and DHA present in seafood are incorporated in multiple phospholipid classes (e.g. phosphatidylethanolamine, phosphatidylcholine and plasmalogens), which may have different absorption properties and biological activities than the TAG and ethyl ester forms present in refined fish oils and medications ${ }^{(36,37)}$. Seafood also contains vitamins, minerals and other nutrients that affect the bioactivities of accompanying fatty acids ${ }^{(38)}$. However, future feeding trials may benefit by providing $n-3$ supplements to participants unwilling or unable to eat seafood on a daily basis in order to provide more uniform amounts of EPA + DHA.

\section{Limitations}

Limitations of $24 \mathrm{~h}$ recalls for assessing nutrient intakes include the dependence on self-reported data and under-reporting ${ }^{(39,40)}$. However, multiple $24 \mathrm{~h}$ recalls have been validated with doubly labelled water and are more highly correlated with present energy intake than FFQ and diet history questionnaires $^{(2,41-46)}$. The $24 \mathrm{~h}$ diet recall method is considered a valid tool for estimating dietary intake and is frequently used to validate new methods of diet assessment ${ }^{(47,48)}$. In addition, this method allowed us to update the nutrient database for the specific study foods and recipes for a more accurate reflection of study food intake during the diet intervention. The NDSR contains detailed fatty acid data, and our efforts to reconcile the fatty acid contents of studyprovided foods with the NDSR database probably improved the accuracy of the diet recalls.

Some of the low-LA foods recommended for purchase were not able to be reconciled with the NDSR database and, therefore, our $24 \mathrm{~h}$ recall data may overestimate LA intakes during the intervention in both diet groups. Because fatty acid values from direct laboratory testing were reconciled with the NDSR database for all study-provided animal foods, and these study foods had fewer zero values for AA, EPA and DHA than corresponding foods in the NDSR database used in the pre-intervention phase, apparent increases in AA, EPA and DHA may have been overestimated. The observed reduction in erythrocyte LA in both groups and marked increase in erythrocyte EPA and DHA in the H3-L6 group provide further evidence of adherence to the study interventions, but cannot quantify whether the nutrient intake targets were achieved. Finally, because this population with chronic headaches may have been more adherent to the study diets due to the potential for pain relief, the magnitudes of the observed 

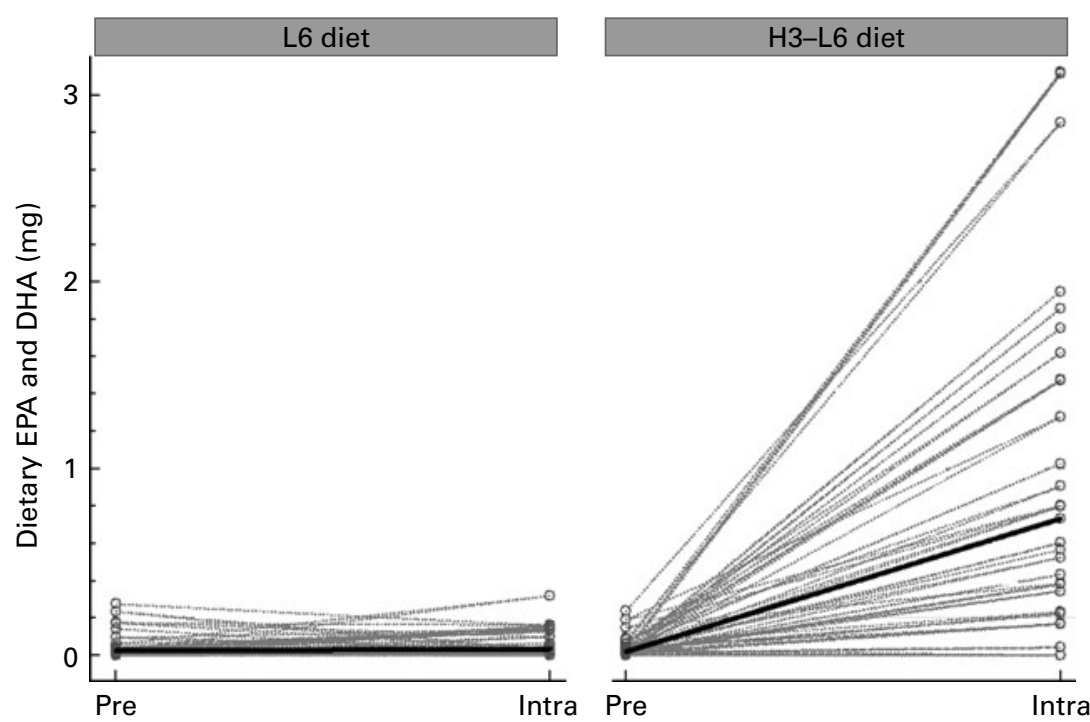

Fig. 2. Dietary EPA+ DHA in the pre-intervention (Pre) and intra-intervention (Intra) periods. L6, low- $n-6$ diet; H3-L6, high- $n-3$ low- $n-6$ diet. - , Median dietary EPA+DHA intake.

dietary changes are not necessarily generalisable to non-pain populations.

\section{Conclusion}

Dietary LA can be lowered to $\leq 2.5 \mathrm{en} \%$, with or without concurrent increases in dietary $n$ - 3 PUFA, in an outpatient clinical trial setting using this integrated diet method. These diet methods are intended to serve as a template for future human trials in other clinical populations to evaluate the effects of lowering dietary LA.

\section{Supplementary material}

To view supplementary material for this article, please visit http://dx.doi.org/10.1017/S0007114512005181

\section{Acknowledgements}

The authors declare that they have no competing interests. The authors would like to thank the following individuals for their research assistance: Marjorie Busby for expertise with study design; Gus Swenson for study food production management; Dietetic Technicians Clarence Mayo, Jim Howerton and Derrick Williams for preparation of study foods; Olafur Palsson, Beth Fowler, Carol Carr, Regina McCoy and Tim McCaskill (programmer) for design and functionality of the study website; Jim Loewke for erythrocyte and nutrient composition analysis and Duk Hyun for nutrient composition analyses; Chanee Lynch for study coordination; and Rebecca Coble, Amit Ringel, Sharon Majchrzak-Hong and Joseph Graciosa for general research assistance. B. A. M. was the lead dietitian, developed the dietary intervention methods, provided nutrition counselling to research subjects and contributed to the initial draft and revisions of the manuscript. C. E. R. was the co-principal investigator and contributed to the hypotheses, grant application, study design and implementation and interpretation of the initial draft and revisions of the manuscript. K. R. F. contributed to the overall trial development and conduct, performed the statistical analysis and revised the manuscript. D. Z. assisted with manuscript preparation and statistical analysis. M. M. led the NORC research team in creating custom user recipes, collecting the $24 \mathrm{~h}$ dietary recalls and revised the manuscript. J. R. H. contributed to the study design and interpretation and revised the manuscript. J. D. M. was the co-principal investigator and the primary study clinician and contributed to all aspects of the project. All authors contributed to the intellectual content of the manuscript. The authors gratefully acknowledge funding support for the present trial from the Mayday Fund (primary source); the North Carolina Clinical and Translational Sciences Institute (grant UL1RR025747, NCRR, NIH); the UNC Nutrition Obesity Research Center, CHAI Core and Nutrition Epidemiology Core (grant DK056350, NIDDK, NIH); the UNC Research Fellowship in Complementary and Alternative Medicine (grant T32-AT003378, NCCAM, NIH); and the Intramural Research Program of the National Institute on Alcohol Abuse and Alcoholism, NIH. Its contents are solely the responsibility of the authors and do not necessarily represent the official views of the Mayday Fund or the National Institutes of Health.

\section{References}

1. Agricultural Research Service (2010) Nutrient Intakes from Food. Amounts Consumed per Individual, by Gender and Age. What We Eat in America, NHANES 2007-2008 [US Department of Agriculture, editor]. Washington, DC: US Department of Agriculture, Agricultural Research Service.

2. Blasbalg TL, Hibbeln JR, Ramsden CE, et al. (2011) Changes in consumption of omega- 3 and omega- 6 fatty acids in the United States during the 20th century. Am J Clin Nutr 93, 950-962. 
3. Kuipers RS, Luxwolda MF, Dijck-Brouwer DA, et al. (2010) Estimated macronutrient and fatty acid intakes from an East African Paleolithic diet. Br J Nutr 104, 1666-1687.

4. Legrand P, Schmitt B, Mourot J, et al. (2010) The consumption of food products from linseed-fed animals maintains erythrocyte omega-3 fatty acids in obese humans. Lipids 45, 11-19.

5. Mohrhauer H \& Holman RT (1963) Alteration of the fatty acid composition of brain lipids by varying levels of dietary essential fatty acids. I Neurochem 10, 523-530.

6. Mohrhauer H \& Holman RT (1963) The effect of dietary essential fatty acids upon composition of polyunsaturated fatty acids in depot fat and erythrocytes of the rat. $J$ Lipid Res 4, 346-350.

7. Mohrhauer H \& Holman RT (1963) The effect of dose level of essential fatty acids upon fatty acid composition of the rat liver. J Lipid Res 4, 151-159.

8. Bourre JM, Piciotti M, Dumont O, et al. (1990) Dietary linoleic acid and polyunsaturated fatty acids in rat brain and other organs. Minimal requirements of linoleic acid. Lipids 25, 465-472.

9. Lands WE, Libelt B, Morris A, et al. (1992) Maintenance of lower proportions of $(n-6)$ eicosanoid precursors in phospholipids of human plasma in response to added dietary (n-3) fatty acids. Biochim Biophys Acta 1180, 147-162.

10. Clark KJ, Makrides M, Neumann MA, et al. (1992) Determination of the optimal ratio of linoleic acid to alpha-linolenic acid in infant formulas. $J$ Pediatr 120, S151-S158.

11. Anonymous (1999) Dietary supplementation with $n-3$ polyunsaturated fatty acids and vitamin $\mathrm{E}$ after myocardial infarction: results of the GISSI-Prevenzione trial. Gruppo Italiano per lo Studio della Sopravvivenza nell'Infarto miocardico. Lancet 354, 447-455.

12. Pradalier A, Bakouche P, Baudesson G, et al. (2001) Failure of omega-3 polyunsaturated fatty acids in prevention of migraine: a double-blind study versus placebo. Cephalalgia 21, 818-822.

13. Thies F, Garry JM, Yaqoob P, et al. (2003) Association of $n-3$ polyunsaturated fatty acids with stability of atherosclerotic plaques: a randomised controlled trial. Lancet 361, 477-485.

14. Goldberg RJ \& Katz J (2007) A meta-analysis of the analgesic effects of omega-3 polyunsaturated fatty acid supplementation for inflammatory joint pain. Pain 129, 210-223.

15. Filion KB, El Khoury F, Bielinski M, et al. (2010) Omega-3 fatty acids in high-risk cardiovascular patients: a metaanalysis of randomized controlled trials. BMC Cardiovasc Disord 10, 24.

16. Ghosh S, Novak EM \& Innis SM (2007) Cardiac proinflammatory pathways are altered with different dietary $n$ - 6 linoleic to $n-3$ alpha-linolenic acid ratios in normal, fat-fed pigs. Am J Physiol Heart Circ Physiol 293, H2919-H2927.

17. Novak EM, Dyer RA \& Innis SM (2008) High dietary omega-6 fatty acids contribute to reduced docosahexaenoic acid in the developing brain and inhibit secondary neurite growth. Brain Res 1237, 136-145.

18. Dayton S, Hashimoto S \& Pearce ML (1967) Adipose tissue linoleic acid as a criterion of adherence to a modified diet. J Lipid Res 8, 508-510.

19. Hodson L, Skeaff CM \& Fielding BA (2008) Fatty acid composition of adipose tissue and blood in humans and its use as a biomarker of dietary intake. Prog Lipid Res 47, 348-380.

20. Ramsden CE, Mann JD, Faurot KR, et al. (2011) Low omega-6 vs. low omega- 6 plus high omega-3 dietary intervention for chronic daily headache: protocol for a randomized clinical trial. Trials 12, 97.
21. Headache Classification Subcommittee of the International Headache Society (2004) The International Classification of Headache Disorders: 2nd ed. Cephalalgia 24, Suppl. 1, 9-160.

22. Bligh EG \& Dyer WJ (1959) A rapid method for total lipid extraction and purification. Can J Biochem Physiol 37, 911-917

23. Schakel SF, Sievert YA \& Buzzard IM (1988) Sources of data for developing and maintaining a nutrient database. $J \mathrm{Am}$ Diet Assoc 88, 1268-1271.

24. Simopoulos AP (2008) The importance of the omega-6/ omega-3 fatty acid ratio in cardiovascular disease and other chronic diseases. Exp Biol Med (Maywood) 233, 674-688.

25. Rett BS \& Whelan J (2011) Increasing dietary linoleic acid does not increase tissue arachidonic acid content in adults consuming Western-type diets: a systematic review. Nutr Metab 8, 36 .

26. Garland M, Sacks FM, Colditz GA, et al. (1998) The relation between dietary intake and adipose tissue composition of selected fatty acids in US women. Am J Clin Nutr 67, 25-30.

27. Kingsbury KJ, Paul S, Crossley A, et al. (1961) The fatty acid composition of human depot fat. Biochem J 78, 541-550.

28. Kingsbury KJ, Heyes TD, Morgan DM, et al. (1962) The effect of dietary changes on the fatty acid composition of normal human depot fat. Biochem J 84, 124-133.

29. Dayton S, Hashimoto S, Dixon W, et al. (1966) Composition of lipids in human serum and adipose tissue during prolonged feeding of a diet high in unsaturated fat. J Lipid Res 7, 103-111.

30. Katan MB, Deslypere JP, van Birgelen AP, et al. (1997) Kinetics of the incorporation of dietary fatty acids into serum cholesteryl esters, erythrocyte membranes, and adipose tissue: an 18-month controlled study. J Lipid Res $\mathbf{3 8}$, 2012-2022.

31. Rahm JJ \& Holman RT (1964) Effect of linoleic acid upon the metabolism of linolenic acid. J Nutr 84, 15-19.

32. Nakamura MT \& Nara TY (2003) Essential fatty acid synthesis and its regulation in mammals. Prostaglandins Leukot Essent Fatty Acids 68, 145-150.

33. Kitson AP, Stroud CK \& Stark KD (2010) Elevated production of docosahexaenoic acid in females: potential molecular mechanisms. Lipids 45, 209-224.

34. Friesen RW \& Innis SM (2010) Linoleic acid is associated with lower long-chain $n-6$ ad n-3 fatty acids in red blood cell lipids of Canadian pregnant women. Am J Clin Nutr 91, 23-31.

35. Brenna JT, Salem N Jr, Sinclair AJ, et al. (2009) Alpha-linolenic acid supplementation and conversion to $n-3$ long-chain polyunsaturated fatty acids in humans. Prostaglandins Leukot Essent Fatty Acids 80, 85-91.

36. Ulven SM, Kirkhus B, Lamglait A, et al. (2011) Metabolic effects of krill oil are essentially similar to those of fish oil but at lower dose of EPA and DHA, in healthy volunteers. Lipids 46, 37-46.

37. Rossmeisl M, Jilkova ZM, Kuda O, et al. (2012) Metabolic effects of $n$-3 PUFA as phospholipids are superior to triglycerides in mice fed a high-fat diet: possible role of endocannabinoids. PLoS One 7, e38834.

38. Umhau JC (2011) Docosahexaenoic acid supplementation and Alzheimer disease. JAMA 305, 672, author reply 673.

39. Hill RJ \& Davies PS (2001) The validity of self-reported energy intake as determined using the doubly labelled water technique. Br J Nutr 85, 415-430.

40. Novotny JA, Rumpler WV, Riddick H, et al. (2003) Personality characteristics as predictors of underreporting of energy 
intake on 24-hour dietary recall interviews. J Am Diet Assoc 103, 1146-1151.

41. Thompson FE \& Byers T (1994) Dietary assessment resource manual. J Nutr 124, 2245S-2317S

42. Jonnalagadda SS, Mitchell DC, Smiciklas-Wright $\mathrm{H}$, et al. (2000) Accuracy of energy intake data estimated by a multiple-pass, 24-hour dietary recall technique. J Am Diet Assoc 100, 303-308, quiz 309-311.

43. Tran KM, Johnson RK, Soultanakis RP, et al. (2000) In-person vs telephone-administered multiple-pass 24-hour recalls in women: validation with doubly labeled water. J Am Diet Assoc 100, 777-783.

44. Trabulsi J \& Schoeller DA (2001) Evaluation of dietary assessment instruments against doubly labeled water, a biomarker of habitual energy intake. Am J Physiol Endocrinol Metab 281, E891-E899.
45. Blanton CA, Moshfegh AJ, Baer DJ, et al. (2006) The USDA automated multiple-pass method accurately estimates group total energy and nutrient intake. J Nutr 136, $2594-2599$

46. Moshfegh AJ, Rhodes DG, Baer DJ, et al. (2008) The US Department of Agriculture automated multiple-pass method reduces bias in the collection of energy intakes. Am J Clin Nutr 88, 324-332.

47. Hebert JR, Ockene IS, Hurley TG, et al. (1997) Development and testing of a seven-day dietary recall. Dietary Assessment Working Group of the Worcester Area Trial for Counseling in Hyperlipidemia (WATCH). J Clin Epidemiol 50, 925-937.

48. Toobert DJ, Strycker LA, Hampson SE, et al. (2011) Computerized portion-size estimation compared to multiple 24-hour dietary recalls for measurement of fat, fruit, and vegetable intake in overweight adults. J Am Diet Assoc 111, 1578-1583. 